\title{
A Rule-Based Expert System for Industrial Training*
}

\author{
Wensheng Liu, Zandong Sun \\ College of Geophysics and Information Engineering, China University of Petroleum-Beijing, Beijing, China \\ Email: \{Liuws89710079, samzdsun\}@yahoo.com.cn
}

Received March 21, 2012; revised April 22, 2012; accepted April 30, 2012

\begin{abstract}
In this paper, a rule-based expert system, named CHECKOP has been presented for industrial training to avoid potential hazards which result from incorrect operating. It also enables a range of operating scenarios for a chemical plant to be depicted by Delta 3D software. The system facilitates the input of operating sequences into the rule-based CLIPS data base. By analysing these operating sequences enables unsafe plant operation to be corrected and the consideration of preventative measures. Operating sequences can be captured and then operation instructions generated automatically.
\end{abstract}

Keywords: Intelligence; Rule-Based; Expert System; Virtual Reality

\section{Introduction}

Hazard that can cause property damage, injury people and fatalities would happen by Mal-operation of a processing plant. The Hazard and Operability Study (or HAZOP) is a standard analysis technique used in the preliminary safety assessment of processing plant. The HAZOP study is a detailed examination, by a group of experts, within a system to determine what would happen if operators were to operate previous normal design mode. The effect of such behavior is then assessed. CHECKOP is an automated batch HAZOP identifi- cation system developed by Loughborough University. CHECKOP contains a rule-based approach which identifies potential hazards to HAZOP analysis [1].

The training system composed of a range of operating scenarios for a chemical plant be build by Delta 3D software [2], to test trainee whether could followed the operate sequence of valves or not. Since valves, pipes and front-end Virtual Reality (VR) dialogue system are usually designed with 3D Max tools to obtain the operate information from trainee, 3D Max tool is feasible to create the VR model, so the system can facilitates the input of operating instructions into CHECKOP.

The expert domain knowledge, such as previous normal design operator sequence of valves, are stored in a knowledge base and are encoded using CLIPS [3]. The CLIPS is abbreviation of C Language Integrated Production System software, it is a Rule-based database.

In order for an operating sequence to be analysed it must be formally represented, instructions written in natural language may be ambiguous. However, machine

*This paper Sponsored by China Scholarship Council, partly being supported by China State 863 plan (2009AA062802). language is incomprehensible to humans, so the captured operating instructions are modeled using CLIPS. Rules which check for incorrect operation leading to hazardous plant states, The use of artificial intelligence techniques of CLIPS can facilitate the representation of knowledge about the problem domain in a more natural and understandable fashion.

\section{CHECKOP System Architecture}

Major VR system consists of some graphics of powers, valves, and pipes drawn by 3D Studio Max, The trainee can navigate through the plant by moving a mouse. When the valve graphic is clicked by middle mouse button, the node of valve graphic will be triggered on high focus, then the CLIPS rules database dispatch a handle to the valve object, if the trainee left-, or right clicks on a graphic of valve at the same time the graphic of valve on high focus, then a message of "Valve State Is ON/OFF" will be sent to the CLIPS, the translation module will interpret the event and translate it into an operating instruction, which is modeled on a formal template. To facilitate communication with CHECKOP the operating instruction is parsed into XML.

CHECKOP's system model consists of three sections:

- A plant configuration model;

- The operating procedures;

- A rule-based system.

\subsection{Plant Configuration Model}

The plant configuration model describes the plant state. CHECKOP employs an object-oriented approach to model plant items, such as, valves, temperatures and pressures. 
This approach is capable of predicting the dynamic behavior of the plant items. Each item of plant is taken from the library of generic unit models, which forms a knowledge-base.

The plant is specified to be in an initially state. The operating instructions act to update the states of the plant items, for example, operating instructions acts to change the ON/OFF state of the valves. For an operating procedure to be analysed by HAZOP, it must be formally represented. Each operating instruction acts to change the state of the plant. The rule-based CLIPS system tests for potential hazards which result from the operating instructions, the operating instruction also can be captured into a XML file. In the end, a text report is produced for a warning against any undesirable situations, see Figure 1.

\subsection{CHECKOP Procedure Representation}

In order to avoid the ambiguities caused by natural language, the operating procedures are composed using a rule-based template representation. The structure of a template is: Action Item Condition:

$$
\begin{aligned}
& \text { operate pump101.pump_drive } \\
& \text { operate valve102 state }=\text { opened } \\
& \text { check valve102 } \text { state }=\text { closed }
\end{aligned}
$$

The "Action" is the operating procedure, e.g. "operate”, “open”, “check”, etc. "Item” is the equipment instance, e.g. pump101, valve101, etc. The "Condition" consisting of: variable and logical operator, template representation describing operating procedures see [4].

\subsection{CHECKOP Rule-Based System}

A rule-based system is suited to represent the ambiguities knowledge required to test for potential hazards. Rules may be easily added, deleted or updated. This allows the rule-based system to be updated if new information becomes available. A rule consists of the structure "If... Then". If the stipulations of a rule's "If" section are met, for example, incorrect operation occurs in the wrong order, the rule is said to be "activated". If the instruction "operate Pump101.pump_drive” is found within the operating procedure, a later instruction "stop Pump101. pump_drive” must also occur within the procedure or this rule will be activated. Applying the HAZOP guideword "No" to the operating procedure could result in the instruction "stop Pump101.pump_drive" to be missing and cause this rule to activate.

\section{An Example Application}

When a supervisor click train button of VR system, a operating consequence through clicking graphic objects is set up, once test button is clicked, a industrial testing procedure is beginning, whenever the trainee left or right clicks on a graphic of valve, a message of "valve101 state change to ON/OFF" is sent to the CLIPS from VR system, left click could create the instruction "open Valve101", and right click could create the instruction “close Valve101". A sequence of operating instructions is captured from the user. The operating procedure created is formatted to the template representation for CHECKOP input. In the end, when the save button is clicked, the operating sequences are saved into a XML file which can be transformed into instructions. It means that operating sequence can be captured and transformed into operation instructions automatically, as while as, the operating instructions also can be analyzed by a HAZOP system.

\section{Conclusions}

This paper describe the system of CHECKOP, which is a HAZOP tool for industrial training, CHECKOP enables operating sequences to be captured, allowing operation instructions to be generated automatically, The VR tool facilitates the input of operating instructions into a rulebased CLIPS system, the CHECKOP, which tests for potential hazards resulting from incorrect operating in

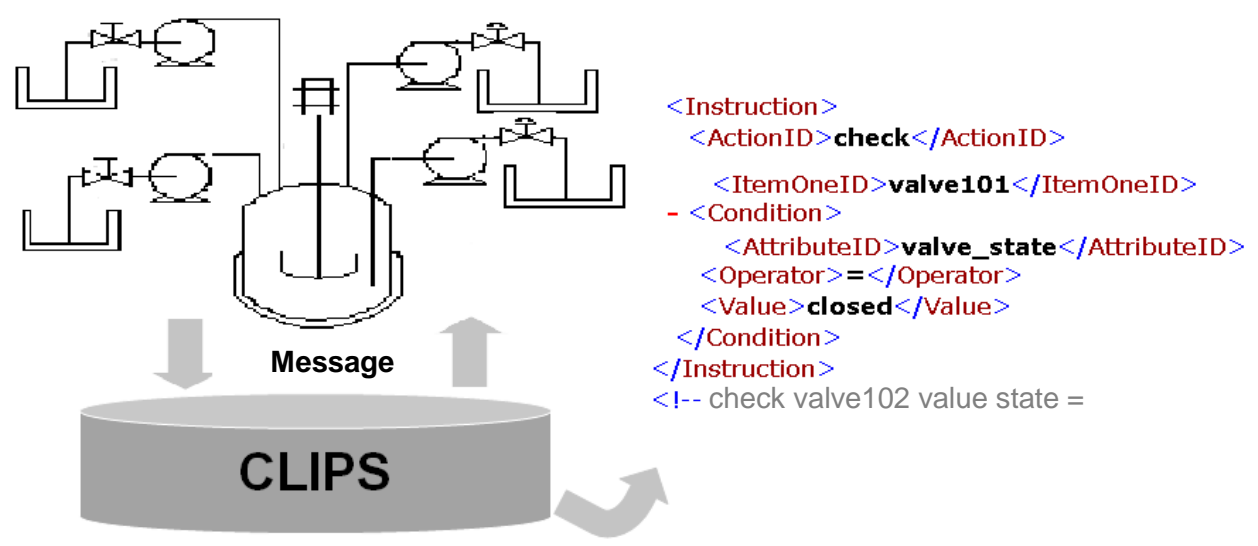

Figure 1. The CHECKOP system model. 
structions. The interaction of the VR tool with CHECKOP allows procedure capture and procedure checking to be integrated.

Rule-based and VR technique provides a flexible way of capturing and specifying operating procedures, and generates operating instructions modeled on a formal template. VR and underlying rule-based CLIPS allow the exact actions as used by the human operator to be captured as the operator interacts with the VR environment. This means that operating sequences can be captured and operation instructions generated automatically, removing the step of having to write the instructions, can provide a fairly convenience interaction between persons and computers.

\section{Acknowledgements}

Mr. LIU Wensheng visited Loughborough university as a academic visitor financial supported by China Scholar- ship Council from 2008 to 2009. During the visit, the author, as a programmer, took part in the procedure of system develop, would also like to thank Prof. Paul for the helpful discussions.

\section{REFERENCES}

[1] F. Mushtaq and P. W. H. Chung, “A Systematic HAZOP Procedure for Batch Processes, and Its Application to Pipeless Plants," Journal of Loss Prevention in the Process Industries, Vol. 13, No. 1, 2000, pp. 41-48. doi:10.1016/S0950-4230(99)00054-6

[2] http://www.delta3d.org

[3] R. Gary, “A Tool for Building Expert Systems,” 2008. http://clipsrules.sourceforge.net/

[4] C. Palmer, P. W. H. Chung, S. A. McCoy and J. Madden, "A Formal Method of Communicating Operating Procedures,” IChemE Symposium Series, Vol. 151, 2006, pp. 448-457. 